\title{
Medical Legal Partnerships: A Key Strategy for Addressing Social Determinants of Health
}

\author{
Heather A. McCabe, JD, MSW and Eleanor D. Kinney, JD, MPH \\ Hall Center for Law and Health, Indiana University School of Law, Indianapolis, IN, USA.
}

J Gen Intern Med 25(Suppl 2):200-1

DOI: $10.1007 / \mathrm{s} 11606-010-1298-9$

(c) Society of General Internal Medicine 2010

$\mathrm{M}$ edical legal partnerships (MLPs), in which lawyers and other professionals engaged in the care of sick patients, are increasing throughout the United States. Since 1993, MLPs have been established at 81 sites. The article by Cohen and colleagues, entitled Medical-Legal Partnership: Using Lawyers to Teach Health Disparities Solutions, provides an excellent overview of the many models of MLPs as well as the role of MLPs in educating fellow professionals about how to address social determinants of health that influence treatment and recuperation. ${ }^{1}$

The theory behind MLPs is that many social and legal problems are involved with a patient's illness and these problems need to be addressed in a multidisciplinary fashion to achieve good and effective care. Medical professionals are taught to get people healthy and send them home. Current reimbursement rules reward hospital stays that are short but do not always prevent readmissions. These approaches are fine so long as the patient returns to a safe environment with adequate help and funds to continue treatment and recuperation. However, that is not always the case.

According to the U.S. Census Bureau, real median household income in the United States was \$50,303 in 2008. The nation's official poverty rate in 2008 was $13.2 \%$ and there were 39.8 million people living in poverty. The number of people without health insurance coverage rose to 46.3 million in 2008, constituting $15.4 \%$ of the population. Also, of the uninsured, $45.9 \%$ had incomes under $\$ 50,000$. Those with lower incomes tended to be single heads of households and of minority status.

Many of these people, because of their lower socio-economic status, have poorer health outcomes and more disease than the more affluent in the population. According to the World Health Organization:

The social determinants of health are the conditions in which people are born, grow, live, work and age, including the health system. These circumstances are shaped by the distribution of money, power and resources at global, national and local levels, which are themselves influenced by policy choices. The social determinants of health are mostly responsible for health inequities-the unfair and avoidable differences in health status seen within and between countries. ${ }^{2}$

Research has shown that some diseases can be treated, but retriggered upon return to the home environment. ${ }^{3,4}$ Indeed, social determinants of health can result in a kind of "revolving door" in which patients are hospitalized multiple times in an episode of illness leaving both the patient and the medical provider frustrated and the achievement of desirable health outcomes illusive.

Without MLPs, physicians, other health professionals and also hospitals do not always have the tools to assist with the home environments. Medical social workers advocate for patients but they are underfunded and often advocacy is not enough. With few tools to tackle the issues of the social determinants of health affecting their patients, physicians are reluctant to screen issues for which they cannot address effectively. , $6^{5}$

MLPs can bridge this gap, particularly when the patient has legal problems that impede treatment and recovery. When physicians screen for issues such as lead paint in the home, access to health insurance and other funds, food security, transportation, and other similar social questions, those working in MLPs have a ready source for referral. Evaluation of some MLPs, spearheaded by the National Center for Medical Legal Partnerships in Boston, MA, indicates that programs are making a difference in the lives of patients by creating improved patient outcomes. Equally important is the change in the practice of health care from a strictly medical model, to a multidisciplinary model.

A critical function of MLPs, as Cohen and colleagues maintain, is educating physicians, social workers, and other professional staff about potential legal issues. The Peninsula Family Advocacy Program (FAP), highlighted by Cohen and colleagues, exemplifies a multidisciplinary approach with the development of an interdisciplinary course for law, medical, and social work students. ${ }^{1}$ Some of the MLPs examined in the article of Cohen and colleagues not only train physicians in practice, but train residents in teaching hospitals. The early training of the professionals involved in working on health care disparities will only serve to make interdisciplinary advocacy for patients a natural part of the work of these professionals.

The basic components of an MLP consist of a medical professional and attorney who work together to address patient concerns. Most MLPs, however, utilize a variety of professions including social workers, paralegals, and/or networks of legal service or pro bono providers. The possible variations on the design are limited only by the resources and imaginations of the participating members.

Innovative MLP models are already being developed. Marion County Health Department in Indianapolis, Indiana has created one MLP run by the local health department in connection with a local legal services agency. The Health Law Partnership (HeLP) at Georgia State University Law in collaboration with Atlanta Legal Aid Society, and Children's Healthcare of Atlanta, Inc. created a law school clinic. Law students provide direct legal services for patients. It is likely there are additional models for MLPs not yet explored. With the leadership of the National Center for Medical 
Legal Partnerships, assistance is available to evaluate MLPs to ensure that any model serves both the service needs of the patient and the educational needs of the professionals involved.

There is much work to do to continue to integrate medical legal education on social determinants of health into the educational curricula of the health professional schools and also law schools. Issues of reimbursement for schools, accreditation standards of professional schools, and professional culture can all be barriers to these types of collaborations. None of these barriers is insurmountable. These interdisciplinary discussions may lead to further innovations to ameliorate the effects of social disparities for patients.

Corresponding Author: Eleanor D. Kinney, JD, MPH, Hall Center for Law and Health, Indiana University School of Law, 530 W. New York Street, IH 136, Indianapolis, IN 46202, USA (e-mail: ekinney@ iupui.edu).

\section{REFERENCES}

1. Cohen E, Fullerton DF, Retkin R, et al. Medical-legal partnership: collaborating with lawyers to identify and address health disparities. J Gen Intern Med. 2010 (in press).

2. World Health Organization. Social Determinants of Health, available at http://www.who.int/social_determinants/en/ (visited Feb. 2, 2010).

3. Zuckerman B, Sandel M, Smith L, Lawton E. Why pediatricians need lawyers to keep children healthy. Pediatrics. 2004;114(1):2248. 224 .

4. Wood PR, Smith LA, Romero D, Bradshaw P, Wise PH, Chavkin W. Relationships between welfare status, health insurance status, and health care among children with asthma. Am J Public Health. 2002;92(9):144652. 1446.

5. Lawton EM. The family advocacy program: a medical-legal collaborative to promote child health and development. Mgmt. Info. Exch. J. 2003 at 14-5.

6. Tyler ET. Allies not adversaries: teaching collaboration to the next generation of doctors and lawyers to address social inequality. J Health Care Law Policy. 2008;11:249-94. 